\title{
Situación profesional y entorno laboral del instructor de Ciclo Indoor Professional situation and labor environment of the Indoor Cycling instructor
}

\author{
*Nerea Estrada-M arcén, *Álvaro Soler-Gracia, *Jaime Casterad-Seral, **Lourdes Cid-Yagüe \\ *U niversidad de Zaragoza (España), **Universidad Autónoma de Madrid (España)
}

\begin{abstract}
Resumen. El Ciclo Indoor (C.I.) es una de las actividades con mayor demanda en la actualidad, y en lasúltimas décadas se ha implantado en la mayoría de los centros de Fitness y gimnasios de gran parte del mundo. Nuestro objetivo es conocer el perfil profesional del instructor de C.I. en España, así como aspectos relacionados con su metodología de trabajo y sus condiciones laborales. Los 57 participantes que conformaron la muestra y que procedían de diferentes lugares de España, ocupaban el puesto de instructor de C.I. en el momento delainvestigación, siendo encuestadosa partir de un instrumento diseñado ad hoc. Se ha empleado una metodología cuantitativa de corte descriptivo. Aunque no podemos constatar que sea una muestra representativa, los datos apuntan a que su perfil es el de un varón de aproximadamente 35 años, con más de 5 años de experiencia, que imparte también otras actividades dirigidas, y trabaja por cuenta ajena. Imparte entre 6 y 7 sesiones de C.I. semanales y existe gran disparidad en cuanto a su nivel y tipo de formación.
\end{abstract}

Palabras clave: Ciclo indoor, Instructor, Ciclismo, Fitness

\begin{abstract}
Indoor Cycling (I.C.) is one of the activities with the greatest demand now adays, and in recent decades, it has been implemented in most fitness centers and gyms in a large part of the world. 0 ur objective is to know the I.C. instructor's professional profile in Spain, as well as other aspects related to their work methodology and working conditions. The respondents were 57 instructors from different parts of Spain (the country), all of them occupying the position of I.C. instructors. They were surveyed based on an instrument designed ad hoc. A quantitative cutting methodology has been used. Although it is not a representative sample, the data suggest that its profile is that of a male of approximately 35 years old, with more than 5 years of experience, who al so teaches other directed activities, and works for others. He teaches between 6-7 sessions of C.I. weekly. There is great disparity in terms of their level and type of training
\end{abstract}

Keywords: Indoor Cycling, Instructor, Fitness.

\section{Introducción}

El mercado global deportivo ha tenido durante las últimas décadas un crecimiento moderado y constante. Y si nos referimos de forma concreta a la industria global de clubes de actividad física relacionada con la salud, ésta actualmente goza de muy buena salud. La Asocia ción Internacional de Clubes de Deportes, Raquetas y Deportes, IHRSA por sus siglas en inglés (Walsh, 2017) reporta que a nivel mundial, en el año 2016, más de 200.000 clubes dieron servicio a unos 162 millones de usuarios. Esta industria del fitness y de la actividad física vinculada con la salud tiene buenas posibilidades de crecimiento, dado que los 10 principales mercados del mundo en este sector representan el $71 \%$ de los ingresos globales de esta industria, lo que evidencia la focalización actual de este mercado.Y en Europa, el crecimiento está impulsado por un aumento de un 3,2\% en el número de clubes, experimentando en esta indus-

\footnotetext{
Fecha recepción: 04-09-20. Fecha de aceptación: 03-02-21 NereaEstrada-Marcén nereaes@ unizar.es
}

tria un crecimiento en el año 2017 con respecto al año anterior de casi un 2\% (EURO PEACTIVE, 2018). Con estos datos, exceptuando los últimos meses caracteriza dos por la incidencia del COVID-19, se puede hablar en losúltimos años de un auge de las actividades de Fitness. Hoy en día, gimnasios y centros privados son un gran negocio global, y en un periodo de tiempo bastante corto, el número de gimnasios, clubes de fitness privados, franquicias, revistas internacionales de fitness y recursos humanos relacionados con el sector ha sufrido un crecimiento drástico (García, 2011; Stern, 2011). Además, se ha experimentado un gran incremento de puestos de trabajo vinculados a todo ello (Estrada-Marcén, Sanz-Gonzalo, Casterad-Seral, Simón-Grima \& RosoM oliner, 2019; M ercader, 2015). Y España cuenta con características específicas con respecto al tipo de insta laciones, a los equipamientos utilizados, a las activida des y servicios ofertados, y a las características de los profesionales que trabajan en este ámbito del Fitness.

Una de las actividades con mayor demanda en la actualidad y que en las últimas décadas se ha implantado en la mayoría de los centros de Fitness y gimnasios de gran parte del mundo es el Ciclo Indoor (C.I.) (Barba 
do, 2011). En el estudio de Juan-Llamas (Juan-Llamas, 2015) se entrevistó a 111 instructores de fitness de diferentes centros en España, y éstos situaron al C.I. como la segunda actividad más impartida en los centros de fitness, suponiendo el $13 \%$ del total de actividades rea lizadas en ellos. El C.I. es un entrenamiento cardiovascular grupal, realizado sobre una bicicleta estática, ejecutada al ritmo de la música. La sesión de C.I. está dirigida por un instructor que marca la intensidad objetivo en cada parte del entrenamiento (Barbado \& Barranco, 2007). Se trata de una disciplina del fitness que nace en EEUU en el año 1992, creada por el exciclista J onathan Goldberg (Barbado, 2011). Se ha consolidado entre las actividades dirigidas de fitness, y su llegada causó en su momento una revolución, puesto que supuso el banderazo de salida para que la práctica del ciclismo fuese más segura y se pudiese realizar durante todo el año debido a las condiciones adversas de la climatología, además de convertirse en una actividad amena y atractiva con la que desarrollar un trabajo cardiovascular (Barbado \& Barranco, 2007). Años después de su implantación, esta actividad está cada vez más profesionalizada, y la formación del instructor cobra un papel fundamental.

Pero a pesar del aumento de profesionales dedica dos a las actividades de fitness en general y al C.I. en particular, hay escasos estudios destinados a describir la situación laboral de los trabajadores de este sector (Boned, Felipe, Barranco, G rimal di-Puyana\& C rovetto., 2015; Campos, M artínez del Castillo, M estre $\&$ Pablos, 2007; Campos, 2005; Estrada-M arcén et al., 2019a), y la mayoría de estos trabajos realizan un análisis global de los profesionales del sector, o emplean agrupaciones poco específicas (Boned et al., 2015), por lo que, en nuestro país, no encontramos estudios previos que se centren de forma específica en la figura del instructor de C.I. . La mayoría de los trabajos que abordan de forma concreta esta actividad dirigida, tratan de dilucidar cuestiones relativas a aspectos como la intensidad de trabajo (Battista, Foster, Andrew, W right, Lucía \& Porcari, 2008; Piacentini, Gianfelici, Faina, Figura y Capranica, 2009), el grado de satisfacción de los usua rios(Bodet, 2006; Lofshult, 2003; Luna\& M undina, 1998; Morente, Yaques \& Zabala 2015; Papadimitriou \& Karteroliotis, 2000; Sanz, Redondo, Gutierrez \& Cua drado, 2005), el gasto energético (Francis, W itucki \& Buono, 1999), o aspectos relacionados con la hidratación (Kang, Chaloupka, Mastrangelo, Hoffman, Ratamess, $\& 0$ 'Connor, 2005).

En relación a la figura del instructor de C.I., consi- deramos que es de importancia dilucidar aspectos relacionados con su formación y sus condiciones de trabajo, puesto que éstos pueden tener influencia directa en su praxis. Su tarea es de responsabilidad, puesto que las sesiones de C.I. suelen ser de alta intensidad, y diversos estudios han demostrado que se queman cantidades significativas de calorías (Ainsworth, Haskell, W hitt, Irwin, Swartz, Strath, 0 Brien, Bassett, Schmitz, \& Emplaincourt, 2000), que hay grandes pérdidas de líquidos (Hazelhurst \& Claassen, 2006), y que las temperaturas corporales sufren grandes aumentos (RamosJiménez, Hernández-Torres, Wall-M edrano, TorresDurán, Juárez-O ropeza, Viloria \& Villalobos-M olina, 2014). Estas condiciones de práctica reúnen factores de riesgo para el desarrollo de diversos problemas de salud o lesiones, como podríaser el caso de la rabdomiolisis (Brogan, Ledesma, Coffino, \& Chander, 2017), por citar algún ejemplo. Si además tenemos en cuenta que dentro de los protocolos de seguridad hay muchos centros de Fitness que no exigen a los futuros clientes un reconocimiento médico como requisito de acceso (Estrada-M arcén, Sanz-Gonzalo, Simón-Grima, Casterad-Seral \& Roso-Moliner, 2019), aún resulta de mayor interés conocer el perfil de los instructores que imparten la actividad y de aspectos relacionados con el desarrollo de su labor profesional. Consideramos que el instructor de C.I. tiene unas características particulares con respecto a otros instructores de actividades de fitness dirigidas. Creemos que debería poseer una formación oficial que le permitiese programar sesiones que cumplan los objetivos de entrenamiento planteados de forma segura y amena, así como formación específica para controlar aspectos propios de la actividad, puesto que inciden directamente en la salud del deportista.

El propósito de este trabajo es conocer el perfil profesional del instructor de C.I. en España en el momento actual, abordando aspectos relacionados con su metodología de trabajo y condiciones laborales, teniendo en cuenta para ello su formación, situación profesional, aspectos contractuales o relacionados con la instalación en la que realiza su actividad, con el fin de establecer un punto de partida en el cual se pueda reivindicar un perfil profesional adecuado en el que se promuevan actividades controladas por personas expertas en el ámbito de la salud. También podría suponer un punto de partida para que los contratadores puedan tener unas orientacionesmínimas; teniendo en cuenta queel objetivo principal de la actividad suele ser mejorar la calidad de vida relacionada con la salud de las personas que la practican, la idoneidad en la contratación de personal cualificado 
para este tipo de actividades es un factor fundamental a tener en cuenta, querepercutiría de forma positivatanto en la empresa como en los clientes.

\section{Metodología}

\section{D iseño del estudio}

Se ha empleado para llevar a cabo este estudio una metodología cuantitativa de corte descriptivo. Se trata de un estudio transeccional o basado en la muestra (Hernández-Sampieri \& Torres, 2018).

Siguiendo a Campos (Campos et al., 2007) una vez establecidos los objetivos del estudio, se elaboró una entrevista estandarizada por medio de cuestionario; tras ello, se llevó a cabo el proceso de recogida de datos y, posteriormente, se procedió al procesamiento y análisis de la información obtenida.

\section{Instrumentos}

Para la recogida de datos se utilizó un cuestionario auto-administrado, diseñado ad hoc en función de los datos obtenidos tras revisión bibliográfica, y de los objetivos perseguidos. A partir de investigaciones previas similares (Boned et al., 2015; Estrada-Marcén et al., 2019a; García, 2011) se diseñó un primer borrador del instrumento a través de la herramienta Google Formularios, que fue sometido a juicio por parte de dos expertos en el ámbito del Fitness, doctores en Ciencias de laActividad Física y del Deporte. Tras depurar ítems, el cuestionario se difundió a través de diferentes redes sociales y servicios de mensajería.

El cuestionario se diseñó con una presentación previa al visionado de las preguntas, en el que se informaba sobre los criterios de inclusión, la confidencialidad de los datos manteniendo el anonimato de la información aportada, así como sobre las intenciones y futuros usos de los datos obtenidos a través del mismo. Se estableció un margen temporal de 15 días, tras los cuales, si no se había recibido respuesta, se repetía el procedimiento de envío como recordatorio.

El instrumento estaba dividido en cinco secciones: variables sociodemográficas, aspectos relativos a la preparación de la sesión, aspectos relativos a la ejecución de la sesión, instalaciones y materiales y perfil de usuarios. Se realizaron en total 47 preguntas, 45 de ellas cerradas y 2 de respuesta corta, cuya cumplimentación duraba entre 5 y 10 minutos.

\section{Participantes}

La población objeto de estudio eran los instructores en activo desarrollando su actividad impartiendo sesiones de C.I. en al gún gimnasio o centro de Fitness espa ñol. Se pretendía conocer su perfil y aspectos relativos a su situación profesional. Se buscó establecer el tama ño muestral. No resulta una tarea fácil averiguar el número de instructores de C.I. ni contactar con ellos, dado que los puestos de trabajo vinculados al mercado de trabajo de la actividad física y del deporte no se recogen estadísticamente de forma específica, por lo que es complicado conocer su cuantificación, teniendo que deducirse de distintos cál culos, encuestas y estadísticas (Campos, González, Abella \& Sancho, 2012). No hay herra mientas que permitan conocer la dimensión de la población objeto de estudio, no hay censo, ni es fácil estimar el número de trabajadores en general en el sector del fitness, por lo que ha sido imposible determinar la dimensión de una población tan específica como es el conjunto de instructores de C.I. en activo. Existen tra bajos como el de Boned (Boned et al., 2015) que estimaba el número total de profesionales en el sector del Fitness (Técnicos de Sala, Entrenadores Per sonales, Técnicos Especialistas en clases colectivas, DirectoresTécnicos y/ o Coordinadores) pero no especificaba el número de instructores de C.I. que podría haber de forma específica. Por este motivo la estimación que permitiese calcular el tamaño de nuestra muestra a partir de estos datos o de otros estudios que describen a los profesionales del fitness en nuestro país (Campos, 2007; Camposet al., 2007; Estrada-M arcén, 2019a), no hasido posible. De este modo, no podemos afirmar que la muestra empleada sea representativa, ante la imposibilidad de calcular el tamaño de la población de nuestro estudio.

Los criterios de inclusión para la participación en el estudio eran ser mayor de edad y encontrarse impartiendo sesiones de C.I. en el momento de la cumplimentación del cuestionario.

Participaron en este estudio un total de 57 sujetos $(n=57)$ de 22 provincias diferentes de España. Las C omunidades autónomas con mayor representación fueron Madrid y Aragón: un $29.8 \%$ de los participantes ejercía su actividad en la Comunidad de Madrid, seguido por un $17 \%$ en la deAragón. De los57 participantes, 49 eran hombres y 8 mujeres, con una media de edad de 35 años (35+5.2).

\section{Tratamiento de los datos}

Para el procesamiento y tratamiento de los datos, y para la elaboración de gráficos, se emplearon Microsoft O ffice Excel, y el paquete estadístico SPSS versión 22.0. 
Se realizó un análisis de los datos de tipo descriptivo, obteniendo frecuencias y porcentajes, o medias y desviaciones típicas, en función de la naturaleza de los da tos.

\section{Resultados y discusión}

En cuanto a la edad de los participantes en el estudio, el $31.6 \%$, tenía entre 30 y 35 años. El rango de edades osciló entre los 20 y los 51 años. La media de edad se situaba en 35 años, y sólo un instructor superaba los 50 años. Boned et al. (2015) señalan que el perfil sociodemográfico del profesional del fitness español se caracteriza por presentar una edad media de 32 años. Según Alves, da Costa, Castañer, Fernándes y Anguera (Alves et al., 2013), esta media es de 25,4 años, puntualizando que en estos estudios se hacía referencia a cualquier profesional del fitness, no refiriéndose de forma específica a los instructores de C.I. En una actividad como el C.I., que conlleva un alto compromiso fisiológico (Ainsworth et al., 2000; Hazelhurst $\&$ Claassen, 2006; Ramos-Jiménez et al., 2014), y basada en la reproducción de modelos, es lógico que encontremos una edad media y máxima de los profesionales que la imparten no demasiado elevada.

En relación al género, el $86 \%$ de los participantes fueron hombres, frente a tan solo un $14 \%$ de mujeres. Esta desigualdad es algo que no parece reflejarse en la realidad general de los gimnasios y centros deportivos. Boned (Boned et al., 2015), Gallardo y Campos (Ga llardo \& Campos-Izquierdo, 2011) y Alves (Alves et al., 2013), afirman que los puestos de técnicos de clases colectivas se caracterizan por estar ocupados principalmente por mujeres, aunque estos estudios están referidos al ámbito del fitness en general. 0 tro estudio rela tivo a los trabajadores de centros de fitness de la ciudad de Zaragoza (Estrada-M arcén et al., 2019a), muestran gran igualdad numérica entre géneros en la ocupación de puestos, cosa que no parece ocurrir en el caso concreto de la actividad del C.I.

En relación a su experiencia (figura 1), la mayoría lleva siendo instructor de C.I. más de 5 años (56.1\%), y
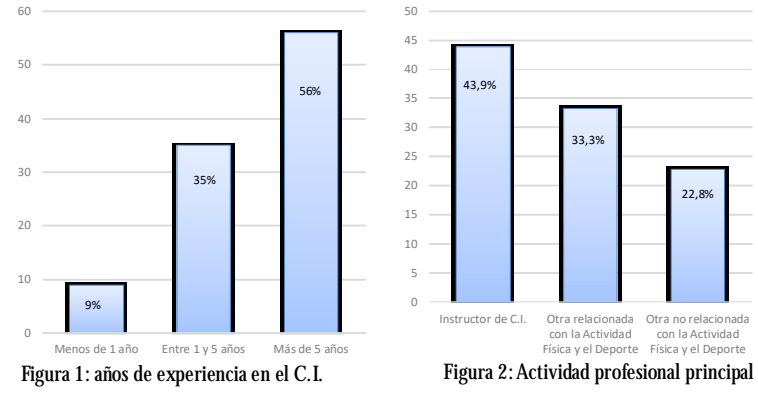

Figura 2: Actividad profesional principal en cuanto a su labor profesional (figura 2) se observa que la fuente principal de ingresos de muchos de los participantes es el C.I. (43.9\%).

En cuanto al volumen de clases, la media de sesiones semanales impartida por cada monitor es de entre 6 y 7 sesiones/ semana, y lamayoríadelosmonitores(36.8\%), imparte entre 2 y 4 sesiones a la semana. El 33.3\%, imparte entre 5 y 9 sesiones. Un $17.5 \%$ de los encuestados, dan más de 11 sesionesa lasemana. Sólo el 3.5\%, imparte menos de 2 sesiones a la semana.

En cuanto a la cantidad de instal aciones en las que el instructor imparte sus sesiones, un $42.1 \%$ las imparte en más de un centro deportivo, mientras que el $57.9 \%$ restante las imparte en un único centro.

Al ser consultados sobre si impartían otras actividades relacionadas con el mundo del fitness, un 33.3\% estaba especializado en C.I., frente a un $66.7 \%$ no especializado. De aquellos que impartían otras actividades, un $20.5 \%$ dirigía además sesiones de actividades coreografiadas, un $79 \%$ actividades de tonificación, un $33 \%$ actividades de Body mind, un 53\% actividades de alta intensidad, y un 33\% otras actividades del ámbito, tal y como se aprecia en la figura 3. El escaso número de sesiones de C.I. semanales impartidas por estos profesionales, junto a este último dato, parece indicar que no es la única actividad a la que dedican su horario laboral. El estudio de Pérez (Pérez et al., 2016) apuntaba a que el ámbito de la actividad física y el deporte se suele caracterizar por la multiocupación y polivalencia de sus trabajadores.

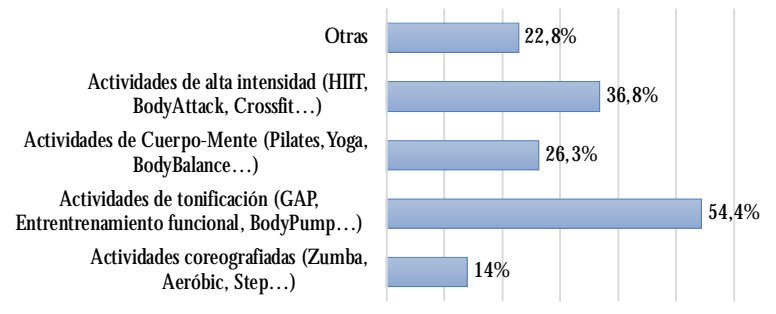

Figura 3: Actividades de Fitness que imparten los participantes, además de C.I.

En cuanto a la formación reglada recibida por los instructores, un $22.8 \%$ de los encuestados dice tener la titulación de graduado/ licenciado en Ciencias de laActividad Física y del Deporte (CCAFD); un 38.6\% posee el título de Técnico en Animación de Actividades Físicas y Deportivas (TAFAD); y el 38.6\% restante no cuenta con una titulación oficial. En el estudio de Boned (Boned et al., 2015) un 11.6\% losinstructores de clases colectivas poseen la licenciatura o el grado en CCAFD, un $11.7 \%$ tienen Magisterio de Educación Física, el $14.1 \%$ poseen la titulación de TAFAD, el $32.7 \%$ son 
Técnicos Deportivos, y un $29.9 \%$ no tiene una titula ción oficial. Cabe resaltar en nuestro estudio el alto porcentaje de trabajadores sin formación oficial, aspecto a considerar si tenemos en cuenta que son instructores que trabajan con personas cuyos objetivos están relacionados en muchos casos con la mejora de la salud. Nos encontramos ante una actividad cuya práctica puede reportar grandes beneficios para la salud (C havarrias, Carlos-Vivas, Collado-Mateo \& Pérez-Gómez, 2019), aunque también puede entrañar ciertos riesgos (Brogan et al., 2017). En ella se hace fundamental una correcta supervisión por parte de profesionales cual ificados, por lo que una buena formación de éstos es un aspecto cla ve. Consideramosque lastitulaciones oficiales (CCAFD, TAFAD y Certificado 0 ficial de Profesionalidad) avalan poseer ciertas competencias necesarias para ser instructor de Actividades Dirigidas, y que las formaciones no oficiales de Ciclo indoor pueden ser de gran interés al dotar al instructor de competencias clave para diseñar, dirigir y supervisar sesiones de esta actividad, siempre que éstas últimas formaciones sean de calidad.

Con respecto a las personas que tienen formación específica en C.I. (no oficial), encontramos una gran heterogeneidad. De esta forma, un $22.8 \%$ se había formado con BestCycling, un 21.1\% con FEDA, un 3.5\% con procycling, y un 3.5\% con Schwinn, siendo más bajo el porcentaje de instructores con otras formaciones como Cycling Room, RPM o Tomahaw.

El 40.4\% de los participantes indica que trabaja para más de una empresa, y el tipo de contrato para ejercer su profesión es principal menteel detrabajador por cuenta ajena $(78.9 \%)$, frente a un $21.1 \%$ de autónomos. En este sector del fitness parece que el contrato por cuenta ajena es el predominante. El estudio de Estrada et al. (Estrada-M arcén et al., 2019a) indicabaque más del 90\% de los profesionales de los centros de fitness de la ciudad de Zaragoza eran contratados por cuenta ajena.

Con respecto a la remuneración, el $42.1 \%$ tenía un sueldo mensual fijo, mientras que un $57.9 \%$ tenía un sueldo mensual fluctuante en función de la actividad realizada. En la figura 4 se pueden observar las cantida des percibidas por cada hora de trabajo. La remunera
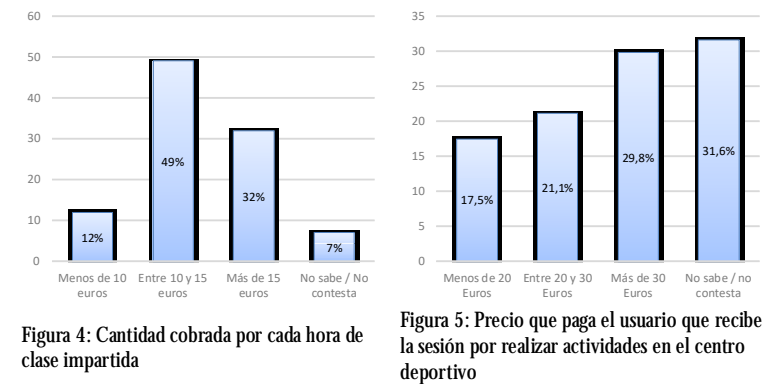

ción percibida no es elevada (10 y 15 euros la hora), por lo que sería lógico encontrar bajo nivel de cualificación profesional.

En cuanto a la cantidad que pagaba el usuario por recibir sus sesiones, había gran diferencia entre los diferentes centros en los que desarrollaban su labor los participantes, como se puede apreciar en la figura 5.

Para comprender con mayor profundidad la complejidad con la que se desarrolla una sesión de C.I. va mos a pasar a referirnos al anál isis de todos los aspectos que intervienen tanto en la preparación, como en la ejecución de la sesión, entendiendo de esta manera la importancia de tener en cuenta factores como la formación de los instructores para mejorar la calidad y seguridad de las mismas.

En cuanto a los aspectos relativos a la preparación de la sesión por parte de instructor, un 93\% de los instructores manejaban un programa de edición musical con regularidad, empleando principalmente MixM eister (92.6\%). Se constató además que un $91.2 \%$ de los instructores encuestados tiene muy en cuenta la velocidad de cada canción (beats por minuto) para confeccionar sus sesiones.

Con referencia al empleo de soporte visual en las sesiones de C.I., la mayoría lo utiliza (63.2\%). De los que lo hacen, la gráfica de esfuerzo es el soporte más utilizado (73.7\%). Un 36.8\% empleavisionado devídeos relacionados con el ciclismo, y un $34.2 \%$ vídeos no rela cionados. M uchos profesionales (86\%) utilizan durante sus sesiones los tres soportes expuestos y un 10.5\% utiliza otro tipo de soporte visual.

A la hora de diseñar una sesión nueva de C.I., el tiempo invertido para hacerlo varía mucho entre monitores, tal como se aprecia en la figura 6. M ás de la mitad de los encuestados, el 56.1\%, asegura invertir más de dos horas en realizar una nueva sesión, una cifra que estimamos muy elevada, sobre todo si la compara mos con los 30-60 minutos que utiliza el $70.3 \%$ de los encuestados en el estudio realizado por Juan-Llamas (Juan-Llamas, 2015) sobre 111 instructores de fitness en general. En todo caso, este dato pone de manifiesto el gran trabajo que existe detrás de la preparación de una sesión de C.I. en comparación con otras actividades del mismo ámbito.

Un $82.5 \%$ de los participantes manifiestan tener en cuenta las características de los usuarios a los que va dirigida la sesión a la hora de diseñarla, y los parámetros que tienen en cuenta para hacerlo son los marcados en la figura 7. Cabe destacar que el $93 \%$ de los encuestados tiene en cuenta para el diseño de la sesión los niveles de 


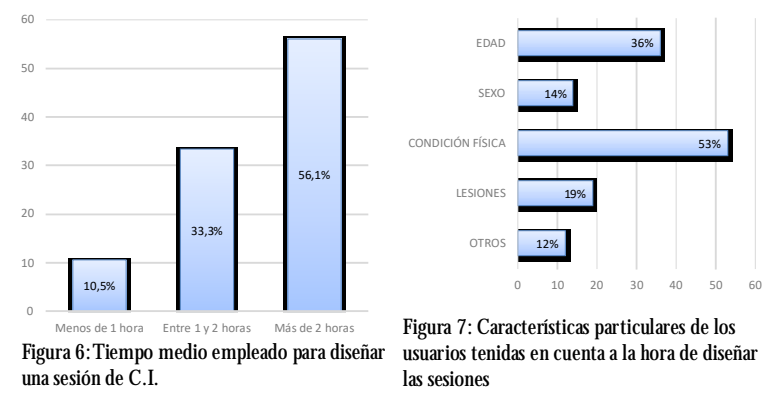

condición física de los participantes, por lo que entendemos que establecen niveles de intensidad en el desa rrollo de sus clases.

Por otro lado, losinstructoresfueron consultadossobre las dinámicas empleadas en sus clases. Un 96.5\% realizaba en ocasiones sesiones temáticas 0 creaba dinámicas diferentes, y un $87.7 \%$ de los monitores tenía en cuenta fechas señaladas como Carnaval, Navidad, Halloween... Normalmente, el usuario es avisado previamente de la realización de dichas sesiones especiales (en un $98.2 \%$ de los casos).

En relación al empleo de sesiones prediseñadas por parte de empresas del sector (Les Mills, Best cycling, ... ) , un 36.8\% utilizan o han utilizado ese sistema, lo que evidencia que la mayoría diseña sus propias sesiones.Y al ser consultados sobre si repiten alguna vez las sesiones con un mismo grupo 0 en un mismo horario, un $42.1 \%$ no lo hace nunca. Un 38.6\% repite la sesión entre 1 y 2 veces al mes, un 17,5\% entre 3 y 4 veces, y únicamente un $1.8 \%$ lo hace más de 4 veces mensuales.

Un 93\% de los instructores determina al confeccionar sus sesiones en qué momentos se pedaleará de pie y en qué momentos se pedaleará sentado. Hay que resaltar que la intensidad de trabajo varía en función de la posición que se adopte sobre la bicicleta. Hay estudios que hablan de un aumento de hasta un $8 \%$ de lafrecuenciacardiaca para unamisma cadencia y resistencia, cuando el sujeto pedalea de pie sobre los pedales, lo que podría ser debido a una mayor necesidad de oxígeno por parte de la musculatura de tren superior en esa posición (Ryschon \& Stray-Gundersen, 1991), por lo que la posición adquirida sobre la bicicleta es un aspecto importante al determinar la intensidad de trabajo, que debe ser tenido en cuenta al diseñar la sesión. Aquellos encuestados que no pensaban en la posición ya desde el diseño de la sesión, manifestaba que variaba la posición en función de la música y el momento (58.8\%), en función de la fatiga que percibía de los usuarios (17.6\%), 0 por otros aspectos no especificados (23.6\%).

Al referirnos de forma específica a la ejecución de la sesión, se les preguntó qué aspectos tenían en cuenta antes de comenzar una sesión. Hay tres aspectos que la gran mayoría de los instructores realizan: preguntar si hay usuarios nuevos (77.2\% de los encuestados); explicar el tipo de trabajo a realizar en la sesión (el 87.7\%), y comprobar que todos los usuarios pueden verle y oírle $(78.9 \%)$. Recordar cómo regular la bicicleta y preguntar si hay alguien con algún problema o lesión, son acciones realizadas antes de la sesión por más del 50\% de los encuestados.Y un $49.1 \%$ hace recomendaciones 0 indicaciones sobre el uso de pulsómetro antes de comenzar.

$Y$ al consultar sobre aquellos aspectos que tienen en cuentadurante laejecución de una sesión, el $98.2 \%$ realiza estiramientos siempre al finalizar, un $94.7 \%$ hace recordatorios sobre la hidratación, un $91.2 \%$ avisa con antelación el cambio de posición. Más del 80\% avisa de la duración de los tiempos de esfuerzo y descanso, motiva a los usuarios en momentos de máxima intensidad y corrige malas posturas y movimientos incorrectos a los usuarios. Lautilización de gestos para incrementar 0 disminuir la resistencia, lo realiza un $71.9 \%$, y corregir el ritmo a aquellos usuarios que no lo siguen, lo realiza el $68.4 \%$ de los instructores encuestados.

En cuanto a los aspectos que los encuestados realizan durante la ejecución de la sesión en referencia al acompañamiento musical vamos a destacar que la mayoría, un $73.7 \%$ de los instructores, llevan en todo momento el ritmo de la música, frente al $19.3 \%$ que en algunos momentos de la sesión no lo lleva.

El $36.8 \%$ de los encuestados deja libertad en algunos momentos a sus alumnos para elegir si llevar o no el ritmo de la música, frente al $29.8 \%$ que busca que lo lleven en todo momento, y tan solo el $8.8 \%$ busca que el alumnado marque el Beat Fuerte con una determinada pierna.

Con respecto a la resistencia de trabajo, un $61.4 \%$, determina siempre el nivel de resistencia que se ha de Ilevar dentro de un rango, frente a un $31.6 \%$ que deja al usuario quien sea el que se regule la resistencia según sensaciones, y una minoría, el 5.3\%, que lo determina solo en subidas y llanos, como podemos observar en la figura 8. La regulación de la resistencia es un aspecto
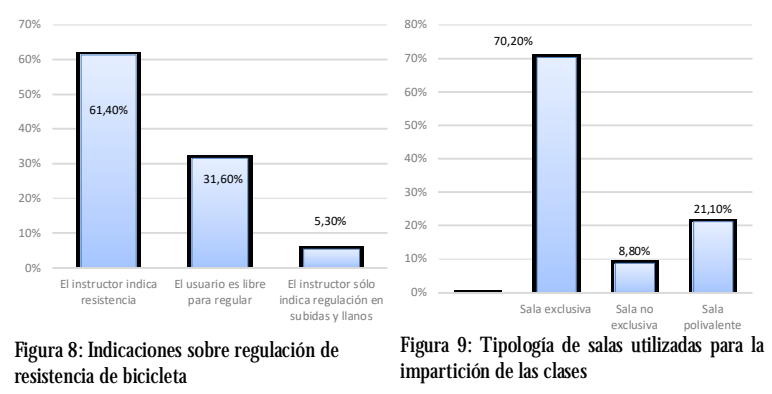
impartición de las clases 
clave al determinar la intensidad de trabajo en C.I., por lo que consideramos que el instructor debe ser capaz de establecer rangos de trabajo adecuados para cada momento de la sesión, estableciendo parámetros de control individualizados.

Hablando de forma concreta de las instalaciones y material, se preguntó a los instructores en relación a los elementos de los que disponen y pueden controlar a nivel audiovisual, de iluminación y de ventilación. Cabe destacar que la inmensa mayoría de los encuestados controlan el volumen delamúsicadesde labicicleta(82.5\%), el $63.2 \%$ utiliza micrófono, y el $57.9 \%$, posee ilumina ción especial en la sala, aunque solo el $36.8 \%$ puede controlarla desde la bicicleta. El $56.1 \%$ dispone de pantalla para proyectar vídeos, y el 33.3\% puede controlarla desde la bicicleta. Un 7\% de los encuestados no posee ninguno de estos elementos. A pesar de que diversos de estos aspectos pueden parecer accesorios, hay investigaciones que demuestran la importancia de algunos de ellos para la mejora de sensaciones subjetivas positivas del participante durante el desarrollo de la sesión (Shaulov \& Lufi, 2009).

Las salas donde se imparten las sesiones (figura 9) suelen ser de uso exclusivo para la actividad de C.I (70.2\%). En un $8.8 \%$ de los casos, la sala no es exclusiva, mientras que un $21.1 \%$ de los instructores imparten sus sesiones tanto en salas de C.I. exclusivas como en salas polivalentes.

Al referirnos a la indumentaria empleada en sus cla ses, prácticamente todos los encuestados utilizan zapa tillas automáticas $(98.2 \%$, ) y la gran mayoría utiliza maillot y culotte $(80.7 \%)$, además de realizar las sesiones con pulsómetro (73.7\%).

En cuanto al tipo de bicicleta empleada en las sesiones, la gran mayoría, el 63.2\%, sigue utilizando bicicletas de freno mecánico sin potenciómetro, frente al $24.6 \%$ que utiliza de freno electromagnético con potenciómetro y el $12.3 \%$ que lo hace con bicicletas de freno electromagnético, pero sin potenciómetro. En referencia al mantenimiento de dichas bicicletas, en el $56.1 \%$ de los casos se realiza entre 1 y 3 veces al año, en el $38.6 \%$ se hace más de 3 veces al año, y sólo un $5.3 \%$ no lo realiza nunca o cuando es necesario por deterioro. Este último aspecto es relevante puesto que, además de ayudar a prolongar la vida útil de la bicicleta, garantiza rá la práctica de la actividad en condiciones de seguridad.

Al ser consultados sobre el perfil de sus usuarios, muchos de los encuestados no trabajan con grupos cerrados en los que siempre asistan las mismas personas
(47.4\%). Esto nos indica que va a ser muy complicado realizar sesiones adaptadas en función de la evolución del grupo, ya que en cada sesión puede haber cambios en sus componentes. Se hará necesario arbitrar estrategias para solventar este aspecto, lo que precisa competencias específicas por parte del monitor.

En referencia al número medio de usuarios por cla se (figura 10), un $59.6 \%$ afirma tener más de 20 usuarios, frente a un $36.8 \%$ que dice tener entre 10 y 20 usuarios, y un 3.5\% que atestigua tener menos de 10 usuarios por sesión, lo que podemos ver en la figura 10. Esta gran afluencia de usuarios a las clases refuerza la importancia de consultar sobre la presencia de nuevos participantes para conocer posibles patologías y darles las indicaciones básicas de posición y manejo de la bicicleta, además de la importancia de realizar una preparación adecuada de las sesiones, teniendo en cuenta posibles adaptaciones a la diversidad. La mayoría de los encuestados afirman que la cantidad de mujeres y hombres que asiste a sus sesiones está equilibrada. $Y$ al consultar sobre los rangos de edad, los instructores destacan que la edad de la mayoría de sus usuarios oscila entre los 30 y los 40 años (63.79\%). Según nuestros instructores, tan solo el $1.72 \%$ de los participantes son mayores de 50 años, lo que podría ser debido a la alta intensidad de la actividad. También podría deberse al perfil tipo del usuario de los centros de fitness; algunos estudios reportan que los mayores de 60 años son socios minoritarios de los centros de fitness (Estrada Marcén et al., 2019b). El citado estudio también indica que la mayoría de los clientes de los centros de fitness de Za ragoza está en una franja entre los 31 y los 45 años.

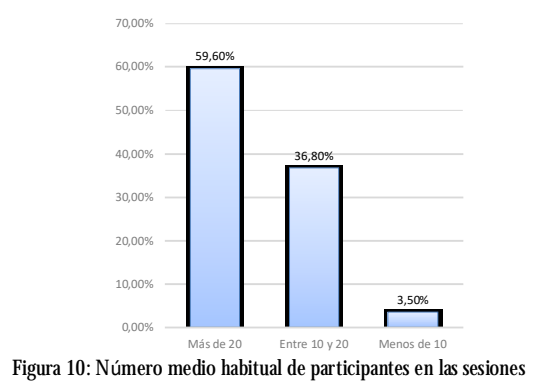

La mayoría de instructores manifiestan con respecto al uso de pulsómetros por parte de los usuarios que sólo entre un $10 \%$ y un $30 \%$ de sus usuarios lo utiliza (43.1\%). La información relativa a este aspecto puede verse en la figura 11. Este es un dato preocupante, ya que el C.I. es una actividad donde se al canzan intensida des altas, por lo que sería conveniente que se utilizara pulsómetro de forma generalizada para poder medir las intensidades de trabajo, para ayudar al usuario a do- 
sificar el esfuerzo, cumplir los objetivos de entrenamiento y trabajar en zonas seguras. El pulsómetro es uno de los aparatos más tradicionales dentro de las tecnologías fitness, y hay estudios en los que se evidencia como la tecnología más empleada por parte de los usua rios de centros de fitnes (Estrada-M arcén et al., 2020).

En referencia a si los instructores recomendaban a sus usuarios realizarse un reconocimiento médico antes de comenzar a realizar la actividad nos encontramos con que el $56.1 \%$ sí lo recomienda, frente a un $43.9 \%$ que no lo hace. A pesar de que parece altamente recomendable, aunque no obligatorio en la actualidad, que los gestores de los centros de fitness exijan un informe médico 0 un certificado médico de aptitud a los usua rios de nuevo ingreso, este requisito no se cumple en muchos casos (Estrada Marcén et al., 2019b).

Si preguntamos a los instructores por el porcentaje de usuarios que creen que está preparado para realizar la actividad a la máxima intensidad con garantías, sus respuestas se presentan en la figura 12 . M uchos de los usuarios no estarían preparados, lo que pone de manifiesto la necesidad de encontrarnos ante profesionales cualificados para diseñar y dirigir la actividad.

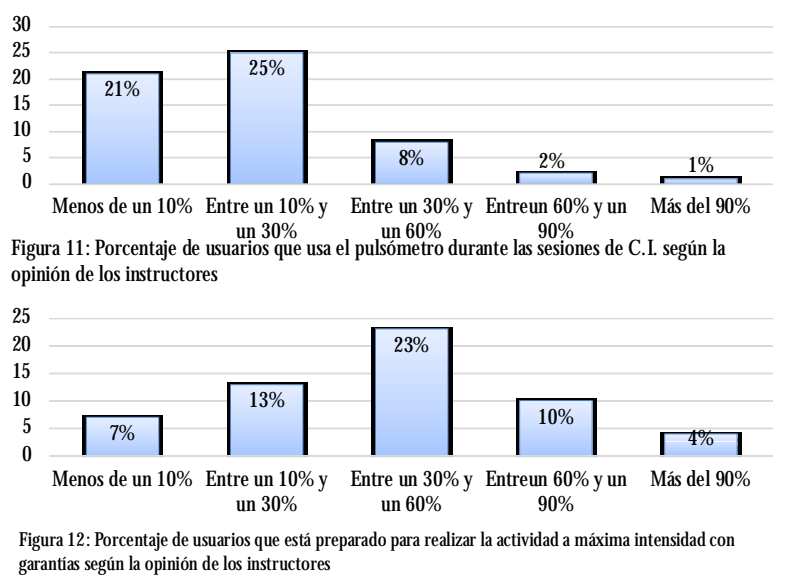

Y al preguntar sobre la motivación de los usuarios, un $45.6 \%$ de los encuestados cree que la mejora de su salud es el motivo principal, seguido de la diversión (42.1\%); tan solo un $7 \%$ de losencuestados opinaquees la mejora del rendimiento deportivo el motivo más relevante, y un 5.3\% opina que es una mezcla de los tres factores anteriores sin anteponer ninguno de ellos. Como podemos observar el porcentaje más alto es la mejora de la salud, por lo que habría que tenerlo en cuenta para programar estas sesiones en función de las necesidades de los usuarios.

\section{Conclusiones}

A pesar de que no se puede afirmar que la muestra sea representativa, debido a la imposibilidad de calcular el tamaño de la población de estudio, los datos parecen apuntar a que el perfil del instructor de C.I. en España es el de un varón de aproximadamente 35 años, con más de 5 años de experiencia en el sector, que imparte también otras actividades relacionadas con el mundo del fitness, y trabaja por cuenta ajena.

Hay un alto porcentaje de instructores de C.I. que no poseen ninguna titulación oficial relacionada ni con dicha especialidad, ni con laActividad Física y el Deporte. Entre las titulaciones no oficiales específicas, encontramos gran variabilidad.

Si tenemos en cuenta la frecuencia con la que se imparten las sesiones, la media de clases que imparte un monitor por semana es de 6.5 y hay un alto porcentaje de instructores que imparten sus sesiones en más de un gimnasio o centro deportivo.

En relación a los aspectos relativos a la preparación de la sesión, casi todos los profesionales necesitan utilizar un programa de edición musical (93\%), y un 63\% prepara soporte visual para utilizar en sus clases, usando fundamentalmente gráficas de esfuerzo. Por todo ello, el tiempo que dicen emplear para confeccionar una nueva sesión de C.I. parece superar el de otro tipo de cla ses del ámbito del fitness. Un 56\% lo fija en dos horas de trabajo por clase, aunque parece que esas sesiones pueden ser utilizadas en más de una ocasión: un 58\% repite su sesión dos o más ocasiones al mes. Casi todos preparan en alguna ocasión sesiones especiales (96\%). La mayoría (67\%) no emplea sesiones prediseñadas por parte de empresas del sector, y todo ello, hace que tengan que invertir una gran cantidad de tiempo y esfuer$z 0$ en la preparación de sus clases sin tener una remuneración adecuada ante tanto esfuerzo.

Antes de comenzar una sesión son tres los aspectos que casi todos los instructores tienen en cuenta: el $88 \%$ de ellos explica el tipo de trabajo que se va a llevar a cabo durante la sesión, el 79\% se preocupan de que todos los usuarios sigan sus consignas y comprueban que todos los usuarios pueden verle y oírle, y el $77 \%$ se preocupan de la orientación de los usuarios noveles, consultando si hay usuarios nuevos en la sala. Por último, podemos afirmar que la gran mayoría de los instructores encuestados tiene en cuenta las características de sus usuarios a la hora de confeccionar una sesión, siendo la condición física y la edad las características más tenidas en cuenta. Estos aspectos hacen evidente la necesidad de una alta cualificación por parte de estos profesionales.

Destacamos la importancia que dan los instructores 
al seguimiento de una higiene postural adecuada, a la realización de los estiramientos al finalizar la sesión, (el $98 \%$ de los instructores reconoce llevarlos a cabo), a la correcta hidratación durante la clase (el 95\% recuerda hidratarse en momentos clave) y al aviso con antelación de un cambio de posición (91\%), lo que permite realizar dicho cambio con garantías de seguridad.

Las salas donde se imparte el C.I. suelen ser exclusivas para la actividad (70\% de los casos), y la mayoría de las bicicletas empleadas son de freno mecánico sin potenciómetro (63\%). Losinstructoresen prácticamente todos los casos usan zapatillas automáticas (98,2\%), y en su mayoría emplean culotte (81\%), indumentarias específicas de la actividad. El pulsómetro tiene un uso alto entre los instructores (74\%).

$Y$ al referirse al perfil de los usuarios, los instructores sitúan su edad principalmente entre los 30 y los 40 años (64\%). El uso de pulsómetros por parte de losusua rios es reducido (lo emplea entre un 10 y un 30\%), y el número de hombres y mujeres que asisten como usuarios a la actividad está bastante equilibrado.

La descripción llevada a cabo en nuestro estudio demuestra la importancia de la formación de los instructores de C.I., dada la gran cantidad de factores a tener en cuenta a la hora de diseñar e implementar las sesiones, y su relación directa con la sal ud de los usuarios que lo practican.

\section{Referencias}

Ainsworth, B. E., Haskell, W. L., W hitt, M. C., Irwin, M. L., Swartz, A. M., Strath, S. J., O Brien, W. L., Bassett, D. R., Schmitz, K. H., \& Emplaincourt, P. 0. (2000). Compendium of physical activities: An update of activity codes and MET intensities. M edicine and science in sports and exercise, 32(9; SUPP/1), S498-S504.

Alves, S. C., da Costa, V. A., Castañer, M., Fernandes, J. J., \& Anguera, M. T. (2013). La conducta de los instructores de Fitness: Triangulación entre la percepción de los practicantes, auto-percepción de los instructores y conducta observada. Revi sta de psicología del deporte, 22(2), 321-329.

Barbado, C. (2011). El ciclo indoor como actividad física saludable. Revista Española de Educación Física y De portes: REEFD, 394, 53-68.

Barbado, C., \& Barranco, D. (2007). M anual de ciclo indoor avanzado. https:/ / dialnet. unirioja. es/ servlet/ libro?codigo $=395424$

Battista, R. A., Foster, C., Andrew, J., W right, G., Lucia,
A., \& Porcari, J. P. (2008). Physiologic responses during indoor cycling. The Journal of Strength $\&$ Conditioning Research, 22(4), 1236-1241.

Bodet, G. (2006). Investigating customer satisfaction in a health club context by an application of the tetraclasse model. European sport management quarterly, 6(2), 149-165.

Boned, C., Felipe, J. L., Barranco, D., Grimal di-Puyana, M., \& Crovetto, M. (2015). Perfil profesional de los trabajadores de los centros de fitness en España. Re vista Internacional de M edicina y Ciencias de la Actividad Física y del Deporte, 15(58), 1-16.

Brogan, M., Ledesma, R., Coffino, A., \& Chander, P. (2017). Freebie rhabdomyolysis: A public health concern. Spin class-induced rhabdomyolysis. The American journal of medicine, 130(4), 484-487.

Campos, A. (2007). Situación profesional de las personas que trabajan en funciones de actividad física y deporte en la Comunidad Autónoma Valenciana (2004). [Http:/ / purl.org/ dc/ dcmitype/ Text, U niver sitat de València]. https:// dialnet. unirioja. es/ servlet/ tesis?codigo $=7194$

Campos, A., M artínez del Castillo, J., Mestre, J. A., \& Pablos, C. (2007). Los profesionales de la organiza ción y gestión de actividad física y deporte en las instalaciones deportivas y entidades: Características socio-demográficas y formativas. RICYDE. R evista Internacional de Ciencias del Deporte, 3(8), 25-38.

Campos, A., González, M. D., A bella, C. P., \& Sancho, J. A. M. (2012). Situación laboral de los entrenadores personales en la Comunidad Valenciana. Aloma: revista de psicologia, ciències de l'educació i de l'esport, 30(1), 167-174.

Campos Izquierdo, A. (2005). Situación profesional de las personas que trabajan en funciones de actividad física y deporte en la Comunidad AutónomaValenciana (2004).

Chavarrias, M., Carlos-Vivas, J., Collado-Mateo, D. , \& Pérez-Gómez, J. (2019). Health Benefits of Indoor Cycling: A Systematic Review. M edicina, 55(8), 452. https:/ / doi.org/ 10.3390/ medicina55080452

Estrada-M arcén, N., Sánchez-Bermúdez, . ., Simón-Grima, J. , \& Casterad-Seral, J. (2020). U so de dispositivos fitness por parte de usuarios de gimnasios. Retos: nuevas tendencias en educación física, deportey recreación, 38, 26-32.

Estrada-M arcén, N., Sanz-Gonzalo, G., Casterad-Seral, J., Simón-Grima, J., \& Roso-M oliner, A. (2019). Perfil profesional de los trabajadores del sector del fitness en la ciudad de Zaragoza. Retos: nuevas tendencias en educación física, deporte y recreación, 35, 185- 
190.

Estrada-M arcén, N., Sanz-Gonzalo, G., Simón-Grima, J., Casterad-Seral, J., \& Roso-M oliner, A. (2019). Loscentrosdefitness delaciudad de Zaragoza. Apunts: Educación física y deportes, 135, 118-136.

EURO PEACTIVE, D. (2018). European Health and Fitness market report. Colonia, Germania: EuropeActive. Disponibile su: http:/ / www. europeactive. eu/ blog/ europeactive-anddel oitte publish-european-health-fitnessmarket-report-2018 [accesso il 08/2018].

Francis, P. R., W itucki, A. S., \& Buono, M. J. (1999). Physiological response to a typical studio cycling session. ACSM 's H ealth \& Fitness Journal, 3(1), 30-36.

Gallardo, J. M., \& Campos-Izquierdo, A. (2011). Situa ción profesional de los recursos humanos de la actividad física y el deporte en el municipio de Coslada. Revista Internacional de M edicina y Ciencias de la Actividad Física y del D eporte, 11(43), 1-1.

García, S. (2011). Características de los centros de fitness de titularidad privada en la Comunidad de M adrid [Http:/ / purl.org/ dc/ dcmitype/ Text, Universidad Europea de Madrid]. https:/ / dialnet. unirioja. es/ servlet/ tesis?codigo $=156386$

Hazelhurst, L. T., \& Claassen, N. (2006). Gender differences in the sweat response during spinning exercise. The J ournal of Strength \& Conditioning R esearch, 20(3), 723-724.

Hernández-Sampieri, R., \& Torres, C. P. M. (2018). M etodología de la investigación (Vol. 4). M cGraw-Hill Interamericana M éxico eD. F DF.

Juan-Llamas, C. (2015). Professional profile of the group classesinstructorsin Spain. AGON International Journal of Sport Sciences, 5(2), 114-125.

Kang, J. I. E., Chaloupka, E. C., Mastrangelo, M. A., H offman, J. R., Ratamess, N. A., \& O 'Connor, E. (2005). Metabolic and perceptual responses during Spinning cycle exercise. Medicine and science in sports and exercise, 37(5), 853-859.

Lofshult, D. (2003). Indoor cycling is still on a roll. (Trends). IDEA H ealth \& Fitness Source, 21(4), 17+.

Luna, R., \& Mundina, J. J. (1998). El marketing estratégico del deporte: Satisfacción, motivación y expectativas. Revista de psicología del deporte, 7(2), 169-174.

Mercader, M. P. L. (2015). Las cual ificaciones profesionales de la familia profesional de actividades físicas y deportivas. Revista Española de Educación Física y De portes, 402, 123-131.

Morente, J., Yaques, P., \& Zabala, M. (2015). Grado de satiffacción del usuario de ciclismo indoor en los centros deportivos de Granada. Retos: nuevas tendencias en educación física, deporte y recreación, 27, 131-135.

Papadimitriou, D. A., \& Karteroliotis, K. (2000). The service quality expectations in private sport and fitness centers: A reexamination of the factor structure. Sport M arketing Quarterly, 9(3), 157-164.

Pérez, M. ,Vilanova, A., \& Grimaldi-Puyana, M. (2016). Mercado actual de trabajo de los graduados en ciencias de la actividad física y el deporte: M irada hacia el autoempleo. Journal of Sports Economics \& Management, 6, 149-162.

Piacentini, M. F., Gianfelici, A., Faina, M ., Figura, F., \& Capranica, L. (2009). Evaluation of intensity during an interval Spinning ${ }^{\circledR}$ session: A field study. Sport Sciences for H ealth, 5(1), 29-36.

Ramos-Jiménez, A., Hernández-Torres, R. P., WallM edrano, A., Torres-Durán, P. V. , Juárez-O ropeza, M. A., Viloria, M., \& Villalobos-Molina, R. (2014). Gender-and hydration-associated differences in the physiological response to spinning. N utricion hospitalaria, 29(3), 644-651.

Ryschon, T.W. \& \& Stray-Gundersen, J. (1991). The effect of body position on the energy cost of cycling M edicine and science in sports and exercise, 23(8), 949-953.

Sanz, I., Redondo, J. C., Gutierrez, P., \& Cuadrado, G. (2005). Lasatisfacción en los practicantes de spinning: Elaboración de una escala parasu medición. European Journal of H uman M ovement, 13, 17-36.

Shaulov, N., \& Lufi, D. (2009). Music and Light during Indoor Cycling. Perceptual and Motor Skills, 108(2), 597-607. https:/ / doi.org/ 10.2466/ pms. 108.2.597607

Stern, M. (2011). Real or rogue charity? Private health clubsvs, theYMCA, 1970-2010. Businessand Economic History, 9, 1-17.

Walsh, K. (2017). IHRSA [International Health, Racquet $\&$ Sportsclub Association] update: Findings from the IHRSA Global Report 2017. H ealth Club M anagement, 7.

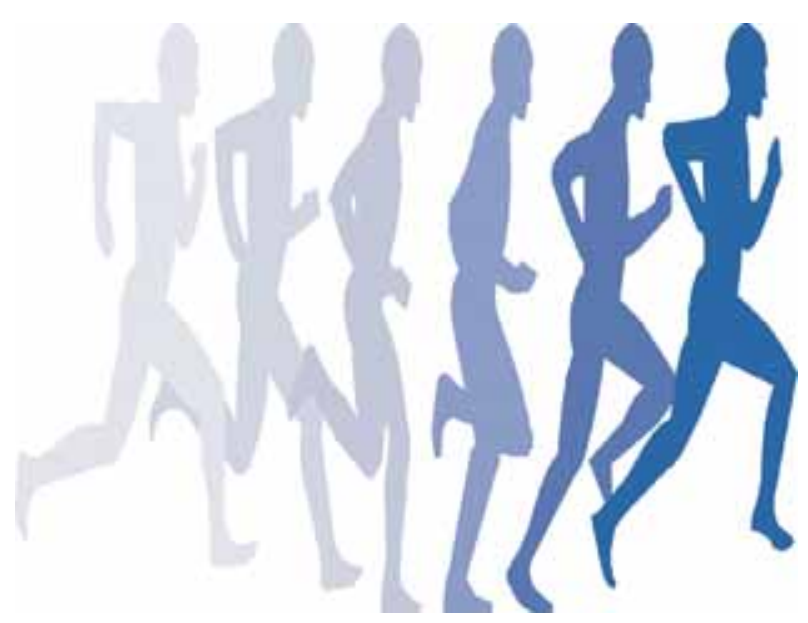

